\title{
Sliding Window Adaptive Fast QR and QR-Lattice Algorithms
}

\author{
Buyurman Baykal, Member, IEEE, and Anthony G. Constantinides, Senior Member, IEEE
}

\begin{abstract}
Sliding window formulations of the fast QR and fast QR-lattice algorithms are presented. The derivations are based on the partial triangularization of raw data matrices. Three methods for window downdating are discussed: the method of plane hyperbolic rotations, the Chambers' method, and the LINPACK algorithm. A numerically ill-conditioned stationary signal and a speech signal are used in finite wordlength simulations of the full QR (nonfast), fast QR, and QR-lattice algorithms. All algorithms are observed to be numerically stable over billions of iterations for double-precision mantissas (53 bits), but as the number of bits is decreased in the mantissa, the algorithms exhibit divergent behavior. Hence, practically, the algorithms can be regarded as numerically stable for long wordlengths.
\end{abstract}

\section{INTRODUCTION}

$\mathbf{S}$ LIDING windows are useful in least-squares estimation in the sense that when a certain number of data points are desired to contribute to the estimation process, unwanted data may be rejected, or old data may be discarded, e.g., in the tracking problem of adaptive filters in nonstationary environments or time-varying systems or reducing the effect of impulsive outliers. On the other hand, the prediction error variance may be higher for sliding windows [1], which highlights the tradeoff between tracking and quality of estimation in adaptive filtering. Window downdating is a delicate step in sliding window algorithms. The "conventional method" plane hyperbolic rotations tend to amplify numerical errors [2]. Bearing this in mind, we discuss alternative methods of window downdating for sliding window fast $\mathrm{QR}$ and $\mathrm{QR}$ lattice algorithms to discover their convergence properties. The most two well-known alternatives to plane hyperbolic rotations are the Chambers' method, which is also known as stabilized hyperbolic rotations [3], and the LINPACK method [4]. It is shown in [5] that these two methods satisfy the "relational stability" condition, which is claimed to help maintain better numerical accuracy, although backward stability [6]-[8] is not guaranteed. We show in the simulations that relational stability may worsen the numerical conditioning of the fast algorithms for short wordlengths. All algorithms developed so far have hyperbolic rotations in their window downdate parts [1], [9], [10]. Some nonfast procedures that relate to the sliding window

Manuscript received June 12, 1996; revised March 5, 1998. The associate editor coordinating the review of this paper and approving it for publication was Dr. Akihiko Sugiyama.

B. Baykal is with the Department of Electrical and Electronic Engineering, Başkent University, Ankara, Turkey.

A. G. Constantinides is with the Department of Electrical and Electronic Engineering, Imperial College of Science, Technology, and Medicine, University of London, London, U.K.

Publisher Item Identifier S 1053-587X(98)07798-8. update of the QR basis matrix also exist [11]-[16]. We assume that the adaptive filtering setup is single-channel and has real inputs.

Consider the input data matrix of dimension $N \times(m+1)$

$$
X_{m+1, N, k}=\left[\begin{array}{llll}
X_{m+1, k} & X_{m+1, k-1} & \cdots & X_{m+1, k-N+1}
\end{array}\right]^{T}
$$

where $X_{m+1, k}=[x(k) \cdots x(k-m)]^{T}$, and let $\boldsymbol{Q}_{m, N, k}$ be such that

$$
\boldsymbol{Q}_{m, N, k} \boldsymbol{X}_{m+1, N, k}=\left[\begin{array}{c}
\mathbf{0} \\
\breve{\boldsymbol{R}}_{m, N, k}
\end{array}\right]
$$

where $\breve{\boldsymbol{R}}_{m, N, k}$ is a lower right triangular matrix of dimension $(m+1) \times(m+1)$, and $\mathbf{0}$ is a null matrix of dimension $(N-m-1) \times(m+1)$. Equation (2) is the basic identity of $\mathrm{QR}$ decomposition, i.e, the raw data is transformed into a triangular matrix, and an orthonormal set of basis vectors is generated in $\boldsymbol{Q}$. Furthermore, assume that at time $k-1, \boldsymbol{Q}_{m, N, k-1}$ is available. To derive a recursive algorithm, we must obtain $\boldsymbol{Q}_{m, N, k}$ from $\boldsymbol{Q}_{m, N, k-1}$ by using new data arrived at time $k$. We also assume that the input signal is a time series and make use of the Hankel structure of the input signal matrix. Hence, the forward prediction of the time series enables us to develop a fast algorithm. Note that no squaring or correlation operations are performed on the input signal $x$ so that the numerical conditioning is not worsened. In fact, $\breve{\boldsymbol{R}}$ is the Cholesky factorization (square-root) of the covariance matrix $R$ of the input signal $x$.

Cioffi [17], together with Regalia and Bellanger [18], have developed the exponentially windowed fast QR algorithm; Proudler et al. [19] and Ling [20] have derived the exponentially windowed fast QR-lattice algorithm. In the sequel, we generalize the QR family to incorporate a sliding window in time. The two algorithms follow the same principles of the QR decomposition, but they are distinguished by the operations on the partially triangularized input data matrix. As for the notation, in general, consistency is maintained with earlier work of Cioffi [1], [17]. First, second, and third subscripts denote prediction order, window length, and discrete-time index, respectively. Table I shows the prediction variables frequently used in the sequel. The symbols $\emptyset$ and $\mathbf{0}$ denote null vectors and null matrices, respectively. In the algorithmic listings, $m / d / s$ denotes $m$ multiplications, $d$ divisions, and $s$ square root operations in terms of complexity. 
TABLE I

Prediction Variables and Notation. The Scalar Variables with Index $m-1$ Run from 0 to $m-1$, AND THE ONES WITH INDEX $m$ RUN FROM 0 TO $m$, EXCEPT FOR $\phi_{m}(k)$, Which RUNS FROM 1 TO $m$

\begin{tabular}{|c|c|c|}
\hline Variables & Forward Prediction & Backward Prediction \\
\hline A posteriori front edge errors & $e_{m, N}(k)$ & $r_{m, N}(k)$ \\
\hline A posteriori rear edge errors & $a_{m, N}(k)$ & $b_{m, N}(k)$ \\
\hline Residual powers & $\alpha_{m, N}(k)$ & $\beta_{m, N}(k)$ \\
\hline Front Likelihood Variable & \multicolumn{2}{|c|}{$\gamma_{m, N}(k)$} \\
\hline Rear Likelihood Variable & \multicolumn{2}{|c|}{$\delta_{m, N}(k)$} \\
\hline Angle normalized front edge errors & $\check{e}_{m, N}(k)=\frac{e_{m, N}(k)}{\gamma_{m, N}^{1 / 2}(k-1)}$ & $\check{r}_{m, N}(k)=\frac{r_{m, N}(k)}{\gamma_{m, N}^{1 / 2}(k)}$ \\
\hline Angle normalized rear edge errors & $\check{a}_{m, N}(k)=\frac{a_{m, N}(k)}{\delta_{m, N}^{1 / 2}(k-1)}$ & $\breve{b}_{m, N}(k)=\frac{b_{m, N}(k)}{\delta_{m, N}^{1 / 2}(k)}$ \\
\hline Power normalized front edge errors & $\bar{e}_{m, N}(k)=\frac{e_{m, N}(k)}{\alpha_{m, N}^{1 / 2}(k)}$ & $\bar{r}_{m, N}(k)=\frac{r_{m, N}(k)}{\beta_{m, N}^{1 / 2}(k)}$ \\
\hline Power normalized rear edge errors & $\bar{a}_{m, N}(k)=\frac{a_{m, N}(k)}{\alpha_{m, N}^{1 / 2}(k)}$ & $\bar{b}_{m, N}(k)=\frac{b_{m, N}(k)}{\beta_{m, N}^{1 / 2}(k)}$ \\
\hline QR lattice variables & $\psi_{m-1, N}^{f}(k)$ & $\psi_{m-1, N}^{b}(k)$ \\
\hline Front edge angles & $\theta_{m-1}^{f}(k)$ & $\theta_{m-1}^{b}(k)$ \\
\hline Rear edge angles & $\vartheta_{m-1}^{f}(k)$ & $\vartheta_{m-1}^{b}(k)$ \\
\hline A posteriori JPE front edge error & \multicolumn{2}{|c|}{$\epsilon_{m, N}(k)$} \\
\hline Angle normalized JPE front edge error & \multicolumn{2}{|c|}{$\check{\epsilon}_{m, N}(k)=\frac{\epsilon_{m, N}(k)}{\gamma_{m, N}^{1 / 2}(k)}$} \\
\hline A posteriori JPE rear edge error & \multicolumn{2}{|c|}{$v_{m, N}(k)$} \\
\hline Angle normalized JPE rear edge error & \multicolumn{2}{|c|}{$\check{v}_{m, N}(k)=\frac{v_{m, N}(k)}{\delta_{m, N}^{1 / 2}(k)}$} \\
\hline QR JPE variable & \multicolumn{2}{|c|}{$\psi_{m-1, N}(k)$} \\
\hline Normalized prediction lattice angle & \multicolumn{2}{|c|}{$\phi_{m}(k)$} \\
\hline Vector of QR forward prediction & \multicolumn{2}{|c|}{$\Psi_{m, N, k}^{f}=\left[\psi_{0, N}^{f}(k) \cdots \psi_{m-1, N}^{f}(k)\right]^{T}$} \\
\hline Vector of QR backward prediction & \multicolumn{2}{|c|}{$\Psi_{m, N, k}^{b}=\left[\begin{array}{lll}\psi_{0, N}^{b}(k) & \cdots & \psi_{m-1, N}^{b}(k)\end{array}\right]^{T}$} \\
\hline Vector of QR JPE & \multicolumn{2}{|c|}{$\Psi_{m, N, k}=\left[\begin{array}{llll}\psi_{0, N}(k) & \cdots & \psi_{m-1, N}(k)\end{array}\right]^{T}$} \\
\hline Vector of front backward normalized errors & \multicolumn{2}{|c|}{$G_{m, N, k}=\left[\begin{array}{llll}\bar{r}_{0, N}(k) & \cdots & \bar{r}_{m-1, N}(k)\end{array}\right]^{T}$} \\
\hline Vector of rear backward normalized errors & \multicolumn{2}{|c|}{$H_{m, N, k}=\left[\begin{array}{llll}\bar{b}_{0, N}(k) & \cdots & \bar{b}_{m-1, N}(k)\end{array}\right]^{T}$} \\
\hline
\end{tabular}

Notation $m$ : Prediction order, $N:$ Window length, $k$ : Discrete time

JPE: Joint Process Extension

\section{Circular And Hyperbolic Rotations}

The QR technique for matrix computations relies on the notion of plane rotations as exemplified in

$$
\left[\begin{array}{c}
\vdots \\
x_{i}^{\prime} \\
\vdots \\
x_{j}^{\prime} \\
\vdots
\end{array}\right]=\left[\begin{array}{lllll}
I & & & & \\
& c & & s & \\
& & I & & \\
& -s & & c & \\
& & & & I
\end{array}\right]\left[\begin{array}{c}
\vdots \\
x_{i} \\
\vdots \\
x_{j} \\
\vdots
\end{array}\right]
$$

where $c^{2}+s^{2}=1$, and all other entries are zero. Such an orthonormal matrix is called a circular rotation matrix, and $c$ and $s$ are the cosine and sine of the rotation angle. Without loss of generality, we assume that cosine variables are on $(i, i)$ and $(j, j)$ entries with $i \neq j$. As a special case, $x_{j}^{\prime}$ can be made zero. This operation is called annihilation of $x_{j}$ pivoted on $x_{i}$. Hence, $x_{j}^{\prime}=0, x_{i}^{\prime}=\sqrt{x_{i}^{2}+x_{j}^{2}}$ and the sine-cosine pair can be solved as

$$
\begin{aligned}
& c=\frac{x_{i}}{\sqrt{x_{i}^{2}+x_{j}^{2}}} \\
& s=\frac{-x_{j}}{\sqrt{x_{i}^{2}+x_{j}^{2}}}
\end{aligned}
$$

which are the fundamental identities of QR decomposition. The position of the minus sign in the transformation can be on either antidiagonal position without loss of generality, depending on the convenience of the development. Such transformations have superior numerical properties in finite precision applications. In QR adaptive filtering, products of 
rotation matrices as in (3) are used, which are also orthonormal.

When $x_{i}^{\prime}$ and $x_{i}$ or $x_{j}^{\prime}$ and $x_{j}$ are swapped in (3) the hyperbolic rotation is obtained

$$
\left[\begin{array}{c}
\vdots \\
x_{i} \\
\vdots \\
x_{j}^{\prime} \\
\vdots
\end{array}\right]=\left[\begin{array}{lllll}
\boldsymbol{I} & & & & \\
& s c & & -t n & \\
& & \boldsymbol{I} & & \\
& -t n & & s c & \\
& & & & I
\end{array}\right]\left[\begin{array}{c}
\vdots \\
x_{i}^{\prime} \\
\vdots \\
x_{j} \\
\vdots
\end{array}\right]
$$

where $s c=1 / c$ and $t n=s / c$ are the secant and tangent of the rotation angle. Unfortunately, hyperbolic rotations tend to amplify finite precision errors [2].

\section{Sliding Window Fast QR-Lattice Algorithm}

The fast QR-lattice algorithm is a reformulation of the leastsquares lattice algorithm to incorporate numerically stable Givens rotations, which provides a more modular structure and set of equations than the fast $\mathrm{QR}$ algorithm by inheriting the modularity of the lattice filter formulation of FIR filtering. There are two alternative ways to derive the $\mathrm{QR}$ decomposition-based adaptive algorithms. The first method in [20] takes the recursive modified Gram-Schmidt algorithm [21] as the starting point and continues to infer the form of the Givens rotations, which constitute the lattice-based algorithm. The second method is based on QR decomposition-based solutions to the forward and backward prediction of the input [19], [22], which we also adopt herein. This approach provides a compact set of equations and a simple interpretation of the QR adaptive filtering. Unlike [19], we do not present generic updates of the variables, and the following derivations are closely tied to [22], which is a special case of [19] (so-called "normal" rotations) in terms of finding the explicit rotations and prediction variables.

The derivation starts by considering the partially triangularized input matrix

$$
\begin{aligned}
& {\left[\begin{array}{ccc}
1 & \emptyset^{T} & 0 \\
\emptyset & \boldsymbol{Q}_{i-1, N-2, k-2} & \emptyset \\
0 & \emptyset^{T} & 1
\end{array}\right]\left[\begin{array}{c}
X_{i+2, k}^{T} \\
\boldsymbol{X}_{i+2, N-2, k-1} \\
X_{i+2, k-N+1}^{T}
\end{array}\right]} \\
& =\underbrace{\left[\begin{array}{ccc}
x(k) & \cdots & x(k-i-1) \\
V^{f} & \mathbf{0} & V^{b} \\
\Psi_{i, N-2, k-1}^{f} & \check{\boldsymbol{R}}_{i-1, N-2, k-2} & \Psi_{i, N-2, k-2}^{b} \\
x(k-N+1) & \cdots & x(k-N-i)
\end{array}\right]}_{S}
\end{aligned}
$$

where $\Psi_{i, N, k}^{f}=\left[\psi_{0, N}^{f}(k) \cdots \psi_{i-1, N}^{f}(k)\right]^{T}$, and a similar definition holds for $\Psi_{i, N, k}^{b}$. The $(N-i-1)$ element vectors $V^{f}$ and $V^{b}$ denote the residual vectors of forward and backward prediction operations. Thus, the input signal matrix is triangularized, except for its first and last columns and rows. The forward and backward prediction of the input signal are based on the annihilation of the input data in the top and bottom rows of $S$ into $\breve{\boldsymbol{R}}$. If the first row from the second element to the $(i+1)$ th element is rotated into $\breve{R}$ with rotations pivoted on $x(k)$, we obtain the forward prediction residual $\breve{e}$ and the backward residual $\breve{r}$, respectively, on the upper left and right corners. ${ }^{1}$ This advances the window length and the time index of variables. Similarly, if the bottom row from the second element to the $(i+1)$ th element is rotated into $\breve{R}$ with rotations pivoted on $x(k-N+1)$, we obtain the rear edge forward prediction residual $\breve{a}$ and backward residual $\breve{b}$, respectively, on the lower left and right corners. In this case, only the window length is advanced. Note that the forward and backward prediction modeling error powers of the algorithm are

$$
\begin{aligned}
\left\|V^{f}\right\|^{2} & =\alpha_{i, N-2}(k-1) \\
\left\|V^{b}\right\|^{2} & =\beta_{i, N-2}(k-1)
\end{aligned}
$$

In addition, the following relations are important in the development

$$
\begin{aligned}
\alpha_{i, N-1}(k) & =\alpha_{i, N-2}(k-1)+\left|\check{e}_{i, N-1}(k)\right|^{2} \\
\alpha_{i, N-1}(k-1) & =\alpha_{i, N-2}(k-1)+\left|\check{a}_{i, N-1}(k-1)\right|^{2}
\end{aligned}
$$

and similar relations for $\beta$. In the $\mathrm{QR}$ decomposition of a shiftinvariant data matrix, forward and backward prediction yield angle-normalized residuals [22], which are defined in Table I.

\section{A. Prediction of the Front Edge of Sliding Window}

Rotation of the bottom and top rows, respectively, via orthonormal matrices $\boldsymbol{Q}^{d}$ and $\boldsymbol{Q}^{u}$, except the side elements of $S$, yields

$$
\begin{aligned}
\boldsymbol{Q}_{i, N, k-1}^{u} \boldsymbol{Q}_{i, N-1, k-2}^{d} \boldsymbol{S} & \\
= & \underbrace{\left[\begin{array}{ccc}
\check{e}_{i, N}(k) & \emptyset^{T} & \check{r}_{i, N}(k-1) \\
V^{f} & \mathbf{0} & V^{b} \\
\Psi_{i, N, k}^{f} & \check{\boldsymbol{R}}_{i-1, N, k-1} & \Psi_{i, N, k-1}^{b} \\
\check{a}_{i, N-1}(k-1) & \emptyset^{T} & \breve{b}_{i, N-1}(k-2)
\end{array}\right]}_{\boldsymbol{N}}
\end{aligned}
$$

where the angle-normalized front forward prediction error $\breve{e}$ and angle-normalized front backward prediction error $\breve{r}$ are generated. Annihilation of all the elements of the last column of $N$ except $\Psi^{b}$ and $\breve{r}$ with an orthonormal matrix $\boldsymbol{Q}_{1}$ pivoted on the element on the top of $\Psi^{b}$ yields, c.f., (7)

$$
\boldsymbol{Q}_{1} N=\left[\begin{array}{ccc}
\check{e}_{i, N}(k) & \emptyset^{T} & \check{r}_{i, N}(k-1) \\
\tilde{V}^{f} & \mathbf{0} & \emptyset \\
\psi_{\boldsymbol{i}, N-1}^{f}(k-1) & \emptyset^{T} & \beta_{\boldsymbol{i}, N-1}^{1 / 2}(k-2) \\
\Psi_{i, N, k}^{f} & \breve{\boldsymbol{R}}_{i-1, N, k-1} & \Psi_{i, N, k-1}^{b} \\
v^{f} & \emptyset^{T} & 0
\end{array}\right] .
$$

The explicit structure of $\boldsymbol{Q}_{1}$ is irrelevant here since it does not affect any of the pertinent variables. The element opposite from the backward prediction error power $\beta^{1 / 2}$ is denoted as

\footnotetext{
${ }^{1}$ The sign denotes the angle normalization of the residuals. Angle normalization means that the residuals are divided by the square roots of the appropriate sample of the front likelihood variable $\gamma$. Similarly, for the prediction of rear edge of the window, the normalization is achieved via division by the square roots of the rear likelihood variable $\delta$.
} 
$\psi^{f}$, which implies an order increase in the $\Psi^{f}$ vector. To find updates on $\check{e}$ and $\psi^{f}$, we rotate $\breve{r}$ by a rotation pivoted on $\beta^{1 / 2}$

$$
\begin{aligned}
{\left[\begin{array}{c}
\check{e}_{i+1, N}(k) \\
\psi_{i, N}^{f}(k)
\end{array}\right]=} & {\left[\begin{array}{ll}
\cos \theta_{i}^{b}(k-1) & -\sin \theta_{i}^{b}(k-1) \\
\sin \theta_{i}^{b}(k-1) & \cos \theta_{i}^{b}(k-1)
\end{array}\right] } \\
& \cdot\left[\begin{array}{c}
\check{e}_{i, N}(k) \\
\psi_{i, N-1}^{f}(k-1)
\end{array}\right]
\end{aligned}
$$

where

$$
\begin{aligned}
& \cos \theta_{i}^{b}(k-1)=\frac{\beta_{i, N-1}^{1 / 2}(k-2)}{\beta_{i, N}^{1 / 2}(k-1)} \\
& \sin \theta_{i}^{b}(k-1)=\check{r}_{i, N}(k-1) \beta_{i, N}^{1 / 2}(k-1)
\end{aligned}
$$

which are obtained similar to (4) with $x_{i}=\beta_{i, N-1}^{1 / 2}(k-$ $2), x_{j}=\check{r}_{i, N}(k-1)$ by using (7c) and (7d) for $\beta$. Generalizing to order $m$, the sequence of transformations above are computed for $i=0, \cdots, m-1$, which completes the forward prediction part of the front edge of the window. The updates of the backward prediction variables are obtained similarly. The first column of $\boldsymbol{N}$ is rotated via an orthonormal matrix $\boldsymbol{Q}_{2}$

$$
\boldsymbol{Q}_{2} \boldsymbol{N}=\left[\begin{array}{ccc}
\check{e}_{i, N}(k) & \emptyset^{T} & \check{r}_{i, N}(k-1) \\
\emptyset & \mathbf{0} & \tilde{V}^{b} \\
\alpha_{i, N-1}^{1 / 2}(k-1) & \emptyset^{T} & \psi_{i, N-1}^{b}(k-1) \\
\Psi_{i, N, k}^{f} & \breve{\boldsymbol{R}}_{i-1, N, k-1} & \Psi_{i, N, k-1}^{b} \\
0 & \emptyset^{T} & v^{b}
\end{array}\right]
$$

where $\psi^{b}$ manifests an order increase in $\Psi^{b}$. Then, $\check{e}$ is annihilated by a rotation pivoted on $\alpha^{1 / 2}$ to obtain the updates

$$
\begin{aligned}
& {\left[\begin{array}{c}
\breve{r}_{i+1, N}(k) \\
\psi_{i, N}^{b}(k)
\end{array}\right]=\left[\begin{array}{cc}
\cos \theta_{i}^{f}(k) & -\sin \theta_{i}^{f}(k) \\
\sin \theta_{i}^{f}(k) & \cos \theta_{i}^{f}(k)
\end{array}\right]} \\
& \cdot\left[\begin{array}{c}
\breve{r}_{i, N}(k-1) \\
\psi_{i, N-1}^{b}(k-1)
\end{array}\right]
\end{aligned}
$$

where

$$
\begin{aligned}
& \cos \theta_{i}^{f}(k)=\frac{\alpha_{i, N-1}^{1 / 2}(k-1)}{\alpha_{i, N}^{1 / 2}(k)} \\
& \sin \theta_{i}^{f}(k)=\frac{\check{e}_{i, N}(k)}{\alpha_{i, N}^{1 / 2}(k)}
\end{aligned}
$$

for $i=0, \cdots, m-1$, which completes the prediction of the front edge of the window.

\section{B. Prediction of the Rear Edge of Sliding Window}

Rotation of the top row via $Q^{u}$ and the bottom row via $\boldsymbol{Q}^{d}$, except the side elements in $\boldsymbol{S}$, yields

$$
\begin{aligned}
\boldsymbol{Q}_{i, N, k-1}^{d} \boldsymbol{Q}_{i, N-1, k-1}^{u} S & \\
= & \underbrace{\left[\begin{array}{ccc}
\check{e}_{i, N-1}(k) & \emptyset^{T} & \check{r}_{i, N-1}(k-1) \\
V^{f} & \mathbf{0} & V^{b} \\
\Psi_{i, N, k}^{f} & \breve{\boldsymbol{R}}_{i-1, N, k-1} & \Psi_{i, N, k-1}^{b} \\
\check{a}_{i, N}(k) & \emptyset^{T} & \check{b}_{i, N}(k-1)
\end{array}\right]}_{\boldsymbol{M}} .
\end{aligned}
$$

The angle-normalized rear forward prediction error $\breve{a}$ and angle-normalized rear backward prediction error $\breve{b}$ are also generated. Going further, the entries in the last column, except $\Psi^{b}$ and $\breve{b}$, are rotated via transformations in $Q_{3}$ pivoted on the entry on top of $\Psi^{b}$

$$
\boldsymbol{Q}_{3} \boldsymbol{M}=\left[\begin{array}{ccc}
\tilde{V}^{f} & \mathbf{0} & \emptyset \\
\psi_{i, N-1}^{f}(k) & \emptyset^{T} & \beta_{i, N-1}^{1 / 2}(k-1) \\
\Psi_{i, N, k}^{f} & \breve{\boldsymbol{R}}_{i-1, N, k-1} & \Psi_{i, N, k-1}^{b} \\
\check{a}_{i, N}(k) & \emptyset^{T} & \breve{b}_{i, N}(k-1)
\end{array}\right] .
$$

The explicit structure of $\boldsymbol{Q}_{3}$ is irrelevant here as in the prediction of the front edge of the window. To obtain an update on $\psi^{f}$ and $\breve{a}$, we annihilate $\breve{b}$ by a rotation pivoted on $\beta^{1 / 2}$, the result of which is given as

$$
\begin{aligned}
{\left[\begin{array}{c}
\psi_{i, N}^{f}(k) \\
\breve{a}_{i+1, N}(k)
\end{array}\right]=} & {\left[\begin{array}{cc}
\cos \vartheta_{i}^{b}(k-1) & \sin \vartheta_{i}^{b}(k-1) \\
-\sin \vartheta_{i}^{b}(k-1) & \cos \vartheta_{i}^{b}(k-1)
\end{array}\right] } \\
& \cdot\left[\begin{array}{c}
\psi_{i, N-1}^{f}(k) \\
\breve{a}_{i, N}(k)
\end{array}\right]
\end{aligned}
$$

where

$$
\begin{aligned}
& \cos \vartheta_{i}^{b}(k-1)=\frac{\beta_{i, N-1}^{1 / 2}(k-1)}{\beta_{i, N}^{1 / 2}(k-1)} \\
& \sin \vartheta_{i}^{b}(k-1)=\frac{\check{b}_{i, N}(k-1)}{\beta_{i, N}^{1 / 2}(k-1)}
\end{aligned}
$$

for $i=0, \cdots, m-1$. Consider again the matrix $M$ to find the updates of backward prediction. Rotate the first column similar to the previous case via an orthonormal matrix $\boldsymbol{Q}_{4}$

$$
\boldsymbol{Q}_{4} \boldsymbol{M}=\left[\begin{array}{ccc}
\emptyset & \mathbf{0} & \underline{r}_{x} \\
\alpha_{i, N-1}^{1 / 2}(k) & \emptyset^{T} & \psi_{i, N-1}^{b}(k) \\
\Psi_{i, N, k}^{f} & \breve{\boldsymbol{R}}_{i-1, N, k-1} & \Psi_{i, N, k-1}^{b} \\
\check{a}_{i, N}(k) & \emptyset^{T} & \breve{b}_{i, N}(k-1)
\end{array}\right] .
$$

Annihilation of $\breve{a}$ pivoted on $\alpha^{1 / 2}$ results in the updates

$$
\begin{aligned}
& {\left[\begin{array}{c}
\psi_{i, N}^{b}(k) \\
\breve{b}_{i+1, N}(k)
\end{array}\right]=\left[\begin{array}{rr}
\cos \vartheta_{i}^{f}(k) & \sin \vartheta_{i}^{f}(k) \\
-\sin \vartheta_{i}^{f}(k) & \cos \vartheta_{i}^{f}(k)
\end{array}\right]} \\
& \cdot\left[\begin{array}{c}
\psi_{i, N-1}^{b}(k) \\
\breve{b}_{i, N}(k-1)
\end{array}\right]
\end{aligned}
$$

where

$$
\begin{aligned}
& \cos \vartheta_{i}^{f}(k)=\frac{\alpha_{i, N-1}^{1 / 2}(k)}{\alpha_{i, N}^{1 / 2}(k)} \\
& \sin \vartheta_{i}^{f}(k)=\frac{\check{a}_{i, N}(k)}{\alpha_{i, N}^{1 / 2}(k)}
\end{aligned}
$$

for $i=0, \cdots, m-1$. Hence, the prediction of the rear edge of the window has been achieved, although it is not clear how to obtain the window downdated variables $\psi^{f}$ and $\psi^{b}$ because they appear on the right-hand sides of transformations. For the sake of clarity, it will be discussed in the next section. The updates of the prediction error powers follow from the relations in (7). 


\section{Window Downdate Rotations}

In the sliding-window fast QR-lattice algorithm outlined above, reshuffling of the variables is needed in the window-downdate relations, namely, (17) and (20), to obtain the window-downdated variables. Hence, $\psi_{N}^{f}, \psi_{N-1}^{f}$ and $\psi_{N}^{b}, \psi_{N-1}^{b}$ are swapped, which modifies the orthogonal transformations to hyperbolic ones, as follows:

$$
\begin{aligned}
& {\left[\begin{array}{c}
\psi_{i, N-1}^{f}(k) \\
\breve{a}_{i+1, N}(k)
\end{array}\right]=\left[\begin{array}{rc}
\sec \vartheta_{i}^{b}(k-1) & -\tan \vartheta_{i}^{b}(k-1) \\
-\tan \vartheta_{i}^{b}(k-1) & \sec \vartheta_{i}^{b}(k-1)
\end{array}\right]} \\
& \text { - }\left[\begin{array}{l}
\psi_{i, N}^{f}(k) \\
\breve{a}_{i, N}(k)
\end{array}\right] \\
& {\left[\begin{array}{l}
\psi_{i, N-1}^{b}(k) \\
\breve{b}_{i+1, N}(k)
\end{array}\right]=\left[\begin{array}{rr}
\sec \vartheta_{i}^{f}(k) & -\tan \vartheta_{i}^{f}(k) \\
-\tan \vartheta_{i}^{f}(k) & \sec \vartheta_{i}^{f}(k)
\end{array}\right]} \\
& \cdot\left[\begin{array}{c}
\psi_{i, N}^{b}(k) \\
\breve{b}_{i, N}(k-1)
\end{array}\right]
\end{aligned}
$$

for $i=0, \cdots, m-1$ similar to (5). We must proceed to obtain the window-downdated variables from (22) and (23), although they contain hyperbolic rotations. On the other hand, the obvious advantages are high modularity of the structure and ease of implementation in parallel processors.

1) Chambers' Method: A reordering of operations in hyperbolic rotations (the so-called Chambers' algorithm [3]) has been proven to satisfy a condition so-called "relational stability" [5]. Rewrite the hyperbolic transformation

$$
\begin{aligned}
& {\left[\begin{array}{rr}
\sec \vartheta & -\tan \vartheta \\
-\tan \vartheta & \sec \vartheta
\end{array}\right]} \\
& =\left[\begin{array}{cc}
1 & 0 \\
-\sin \vartheta & \cos \vartheta
\end{array}\right]\left[\begin{array}{cc}
\sec \vartheta & -\tan \vartheta \\
0 & 1
\end{array}\right]
\end{aligned}
$$

so that the window downdate is performed in two stages. The incurred extra computational cost is two divisions per transformation to compute sine-cosine pairs.

\section{Sliding Window FASt QR Algorithm}

The rationale behind the fast $\mathrm{QR}$ algorithm is to use another set of orthogonal rotations available from the least-squares prediction theory to obtain the backward prediction variables.

\section{A. Prediction of the Front Edge of Sliding Window}

The first step concerning the forward prediction variables is identical to the QR-lattice algorithm. In the estimation of the backward prediction variables, alternatively, the normalized prediction lattice structure provides the following relationship [18]:

$$
\begin{aligned}
& {\left[\begin{array}{c}
\bar{e}_{i-1, N}(k) \\
\bar{r}_{i, N}(k)
\end{array}\right]} \\
& \quad=\left[\begin{array}{cc}
\cos \phi_{i}(k) & \sin \phi_{i}(k) \\
-\sin \phi_{i}(k) & \cos \phi_{i}(k)
\end{array}\right]\left[\begin{array}{c}
\bar{e}_{i, N}(k) \\
\bar{r}_{i-1, N}(k-1)
\end{array}\right]
\end{aligned}
$$

for $i=m, \cdots, 1$, where an overbar denotes the powernormalized variables. Hence, c.f., Table I

$$
\bar{e}_{i, N}(k)=\frac{e_{i, N}(k)}{\alpha_{i, N}^{1 / 2}(k)}=\frac{\check{e}_{i, N}(k) \gamma_{i, N}^{1 / 2}(k-1)}{\alpha_{i, N}^{1 / 2}(k)}
$$

$$
\bar{r}_{i, N}(k)=\frac{r_{i, N}(k)}{\beta_{i, N}^{1 / 2}(k)}=\frac{\check{r}_{i, N}(k) \gamma_{i, N}^{1 / 2}(k)}{\beta_{i, N}^{1 / 2}(k)} .
$$

In addition, the rotation variables are

$$
\begin{aligned}
\sin \phi_{i}(k) & =\frac{\psi_{i-1, N}^{f}(k)}{\alpha_{i-1, N}^{1 / 2}(k)} \\
\cos \phi_{i}(k) & =\frac{\alpha_{i, N}^{1 / 2}(k)}{\alpha_{i-1, N}^{1 / 2}(k)} .
\end{aligned}
$$

The sequence of rotations in (25) start with

$$
\begin{aligned}
\bar{e}_{m, N}(k) & =\frac{\check{e}_{m, N}(k) \gamma_{m, N}^{1 / 2}(k-1)}{\alpha_{m, N}^{1 / 2}(k)} \\
\alpha_{m, N}^{1 / 2}(k) & =\sqrt{\alpha_{m, N-1}(k-1)+\left|\check{e}_{m, N}(k)\right|^{2}}
\end{aligned}
$$

in which $\gamma^{1 / 2}$ is available from the previous iteration. The lower order $\alpha$ 's can be computed from

$$
\alpha_{i-1, N}^{1 / 2}(k)=\sqrt{\alpha_{i, N}(k)+\left|\psi_{i-1, N}^{f}(k)\right|^{2}}
$$

for $i=m, \cdots, 1$ [18]. Last, $\bar{r}_{0, N}(k)=\bar{e}_{0, N}(k)$ because the zeroth-order prediction corresponds to the input signal itself. The updated normalized backward prediction errors become

$$
\begin{aligned}
G_{m, N, k} & =\left[\begin{array}{c}
\bar{r}_{0, N}(k) \\
\bar{r}_{1, N}(k) \\
\vdots \\
\bar{r}_{m-1, N}(k)
\end{array}\right] \\
= & {\left[\begin{array}{l}
\sin \theta_{0}^{b}(k) \\
\sin \theta_{1}^{b}(k) \cos \theta_{0}^{b}(k) \\
\vdots \\
\sin \theta_{m-1}^{b}(k) \cos \theta_{m-2}^{b}(k) \cdots \cos \theta_{0}^{b}(k)
\end{array}\right] . }
\end{aligned}
$$

It is well known that [17], [18] the likelihood variable $\gamma$ can be computed as

$$
\begin{aligned}
\gamma_{m, N}^{1 / 2}(k) & =\prod_{i=0}^{m-1} \cos \theta_{i}^{b}(k)=\sqrt{1-\left\|G_{m, N, k}\right\|^{2}} \\
& =\sqrt{1-\sum_{i=0}^{m-1}\left|\bar{r}_{i, N}(k)\right|^{2}} .
\end{aligned}
$$

A derivation of the above relationships in the QR decomposition framework is also given in [23]. It is convenient to summarize this derivation herein to clarify the development in the window downdate part. The $i$ th-order rotation matrix $Q$ can be constructed iteratively by considering the product

$$
\left[\begin{array}{ll}
1 & \emptyset^{T} \\
\emptyset & \boldsymbol{Q}_{i, N-1, k-1}
\end{array}\right]\left[\begin{array}{c}
X_{i+1, k}^{T} \\
\boldsymbol{X}_{i+1, N-1, k-1}
\end{array}\right]=\left[\begin{array}{c}
X_{i+1, k}^{T} \\
\mathbf{0} \\
\check{\boldsymbol{R}}_{i, N-1, k-1}
\end{array}\right]
$$

Thus, $\boldsymbol{Q}_{i, N, k}$ is obtained through a set of rotations that annihilate the first row of the right-hand side matrix. We can write

$$
\hat{\boldsymbol{Q}}_{k}\left[\begin{array}{cc}
1 & \emptyset^{T} \\
\emptyset & \boldsymbol{Q}_{i, N-1, k-1}
\end{array}\right]=\boldsymbol{Q}_{i, N, k}
$$


The first column of the matrix $\boldsymbol{Q}_{i, N, k}$ is $\left[\gamma_{i+1, N}^{1 / 2}(k) \quad \emptyset^{T}\right.$ $\left.G_{i+1, N, k}^{T}\right]^{T}$. Now, consider

$$
\begin{aligned}
& {\left[\begin{array}{rr}
\boldsymbol{Q}_{i-1, N, k-1} & \emptyset \\
\emptyset^{T} & 1
\end{array}\right]\left[\begin{array}{c}
\boldsymbol{X}_{i+1, N, k} \\
\emptyset^{T}
\end{array}\right]} \\
& =\left[\begin{array}{cc}
\check{e}_{i, N}(k) & \emptyset^{T} \\
V_{1}^{f} & \mathbf{0} \\
\Psi_{i, N, k}^{f} & \check{\boldsymbol{R}}_{i-1, N, k-1} \\
0 & \emptyset^{T}
\end{array}\right] .
\end{aligned}
$$

Let us rotate $V_{1}^{f}$ via $\boldsymbol{Q}_{V_{1}}$ pivoted on the last entry of the first column. Then, annihilate $\breve{e}$ via $\boldsymbol{Q}_{e}$. The first column of the matrix of the right-hand side becomes $\left[\begin{array}{lll}\emptyset^{T} & \Psi_{i, N, k}^{f T} & \alpha_{i, N}^{1 / 2}(k)\end{array}\right]^{T}$. When $\Psi_{i, N-1, k}^{f}$ is rotated via $\boldsymbol{Q}_{\psi}$ pivoted on $\alpha^{1 / 2}$, the triangularization process is completed. The resulting orthonormal matrix can be written as

$$
\left[\begin{array}{ll}
\boldsymbol{I} & \emptyset^{T} \\
\emptyset & \boldsymbol{Q}_{\psi}
\end{array}\right] \boldsymbol{Q}_{e} \boldsymbol{Q}_{V_{1}}\left[\begin{array}{cc}
\boldsymbol{Q}_{i-1, N, k-1} & \emptyset \\
\emptyset^{T} & 1
\end{array}\right]=\boldsymbol{Q}_{i, N, k}
$$

The first columns of both sides are

$$
\left[\begin{array}{ll}
\boldsymbol{I} & \emptyset^{T} \\
\emptyset & \boldsymbol{Q}_{\psi}
\end{array}\right] \boldsymbol{Q}_{e} \boldsymbol{Q}_{V_{1}}\left[\begin{array}{c}
\gamma_{i, N}^{1 / 2}(k-1) \\
\emptyset \\
G_{i, N, k-1} \\
0
\end{array}\right]=\left[\begin{array}{c}
\gamma_{i+1, N}^{1 / 2}(k) \\
\emptyset \\
G_{i+1, N, k}
\end{array}\right] .
$$

Consider the last $N+1$ rows

$$
\boldsymbol{Q}_{\psi}\left[\begin{array}{c}
G_{i, N, k-1} \\
\check{e}_{i, N}(k) \gamma_{i, N}^{1 / 2}(k-1) / \alpha_{i, N}^{1 / 2}(k)
\end{array}\right]=G_{i+1, N, k} .
$$

From (28), we can observe that $\check{e}_{i, N}(k) \gamma_{i, N}^{1 / 2}(k-1) / \alpha_{i, N}^{1 / 2}(k)=$ $\bar{e}_{i, N}(k)$. When the rotations in $\boldsymbol{Q}_{\psi}$ are performed, we obtain the relations in (25). The rotations are defined by the variables in (27).

The explicit structure of the vector in (31) suggest that the individual sine and cosine pairs can be extracted by the following method [18]:

$$
\begin{aligned}
\gamma_{i, N}(k) & =\gamma_{i+1, N}(k)+\left|\bar{r}_{i, N}(k)\right|^{2} \\
\sin \theta_{i}^{b}(k) & =\frac{\bar{r}_{i, N}(k)}{\gamma_{i, N}^{1 / 2}(k)} \\
\cos \theta_{i}^{b}(k) & =\frac{\gamma_{i+1, N}^{1 / 2}(k)}{\gamma_{i, N}^{1 / 2}(k)}
\end{aligned}
$$

for $i=m-1, \cdots, 0$. Thus, the updated sine-cosine pairs for the backward prediction are obtained.

\section{B. Prediction of the Rear Edge of Sliding Window}

The downdate of the forward prediction variables in the fast QR algorithm is identical to the fast QR-lattice algorithm. The sine-cosine pairs corresponding to the backward prediction variables of the rear edge of the window are found similar to (25). This operation can be regarded as prediction of the data to be deleted from the sliding window by using the lattice variables $\psi_{i, N}^{f}(k)$. Now, consider the alternative triangularization of the data matrix

$$
\left[\begin{array}{cc}
1 & \emptyset^{T} \\
\emptyset & \boldsymbol{Q}_{i, N-1, k}
\end{array}\right]\left[\begin{array}{c}
X_{i+1, k-N+1}^{T} \\
\boldsymbol{X}_{i+1, N-1, k}
\end{array}\right]=\left[\begin{array}{c}
X_{i+1, k-N+1}^{T} \\
\mathbf{0} \\
\check{\boldsymbol{R}}_{i, N-1, k}
\end{array}\right]
$$

in contrast to (33). We can obtain an orthonormal matrix $\tilde{Q}$ that triangularizes the data matrix by annihilating the top row. The first column of $\tilde{\boldsymbol{Q}}$ is $\left[\begin{array}{lll}\delta_{i+1, N}^{1 / 2}(k) & \emptyset^{T} & H_{i+1, N, k}^{T}\end{array}\right]^{T}$, where

$$
\begin{aligned}
H_{m, N, k} & =\left[\begin{array}{c}
\bar{b}_{0, N}(k) \\
\bar{b}_{1, N}(k) \\
\vdots \\
\bar{b}_{m-1, N}(k)
\end{array}\right] \\
& =\left[\begin{array}{l}
\sin \vartheta_{0}^{b}(k) \\
\sin \vartheta_{1}^{b}(k) \cos \vartheta_{0}^{b}(k) \\
\vdots \\
\sin \vartheta_{m-1}^{b}(k) \cos \vartheta_{m-2}^{b}(k) \cdots \cos \vartheta_{0}^{b}(k)
\end{array}\right] \\
\delta_{m, N}^{1 / 2}(k) & =\prod_{i=0}^{m-1} \cos \vartheta_{i}^{b}(k)=\sqrt{1-\left.|| H_{m, N, k}\right|^{2}} \\
& =\sqrt{1-\sum_{i=0}^{m-1}\left|\bar{b}_{i, N}(k)\right|^{2} .}
\end{aligned}
$$

Now, consider

$$
\begin{gathered}
{\left[\begin{array}{cc}
\tilde{\boldsymbol{Q}}_{i-1, N, k-1} & \emptyset \\
\emptyset^{T} & 1
\end{array}\right]\left[\begin{array}{c}
X_{i+1, k-N+1} \\
\boldsymbol{X}_{i+1, N-1, k} \\
\emptyset^{T}
\end{array}\right]} \\
=\left[\begin{array}{cc}
\breve{a}_{i, N}(k) & \emptyset^{T} \\
V_{2}^{f} & \mathbf{0} \\
\Psi_{i, N-1, k}^{f} & \check{\boldsymbol{R}}_{i-1, N, k-1} \\
0 & \emptyset^{T}
\end{array}\right]
\end{gathered}
$$

Let us rotate $V_{2}^{f}$ via $Q_{V_{2}}$ pivoted on the last element of the first column, and then, annihilate $\breve{a}$ via $\boldsymbol{Q}_{a}$. The first column of the matrix of the right-hand side becomes $\left[\begin{array}{lll}\emptyset^{T} & \Psi_{i, N, k}^{f T} & \alpha_{i, N}^{1 / 2}(k)\end{array}\right]^{T}$. When $\Psi_{i, N, k}^{f}$ is rotated via $\boldsymbol{Q}_{\psi}$ pivoted on $\alpha^{1 / 2}$, the triangularization process is completed similar to the prediction of the front edge of the window. The resulting orthonormal matrix can be written as

$$
\left[\begin{array}{ll}
\boldsymbol{I} & \emptyset^{T} \\
\emptyset & \boldsymbol{Q}_{\psi}
\end{array}\right] \boldsymbol{Q}_{a} \boldsymbol{Q}_{V_{2}}\left[\begin{array}{cc}
\tilde{\boldsymbol{Q}}_{i-1, N, k-1} & \emptyset \\
\emptyset^{T} & 1
\end{array}\right]=\tilde{\boldsymbol{Q}}_{i, N, k}
$$

The first columns of both sides are

$$
\left[\begin{array}{ll}
\boldsymbol{I} & \emptyset^{T} \\
\emptyset & \boldsymbol{Q}_{\psi}
\end{array}\right] \boldsymbol{Q}_{a} \boldsymbol{Q}_{V_{2}}\left[\begin{array}{c}
\delta_{i, N}^{1 / 2}(k-1) \\
\emptyset \\
H_{i, N, k-1} \\
0
\end{array}\right]=\left[\begin{array}{c}
\delta_{i+1, N}^{1 / 2}(k) \\
\emptyset \\
H_{i+1, N, k}
\end{array}\right]
$$

Consider the last $N+1$ rows

$$
\boldsymbol{Q}_{\psi}\left[\begin{array}{c}
H_{i, N, k-1} \\
\breve{a}_{i, N}(k) \delta_{i, N}^{1 / 2}(k-1) / \alpha_{i, N}^{1 / 2}(k)
\end{array}\right]=H_{i+1, N, k} .
$$

Hence, a similar update to (38) is obtained for the rear edge backward prediction variables. 
TABLE II

Prediction of the Front Edge of the Window

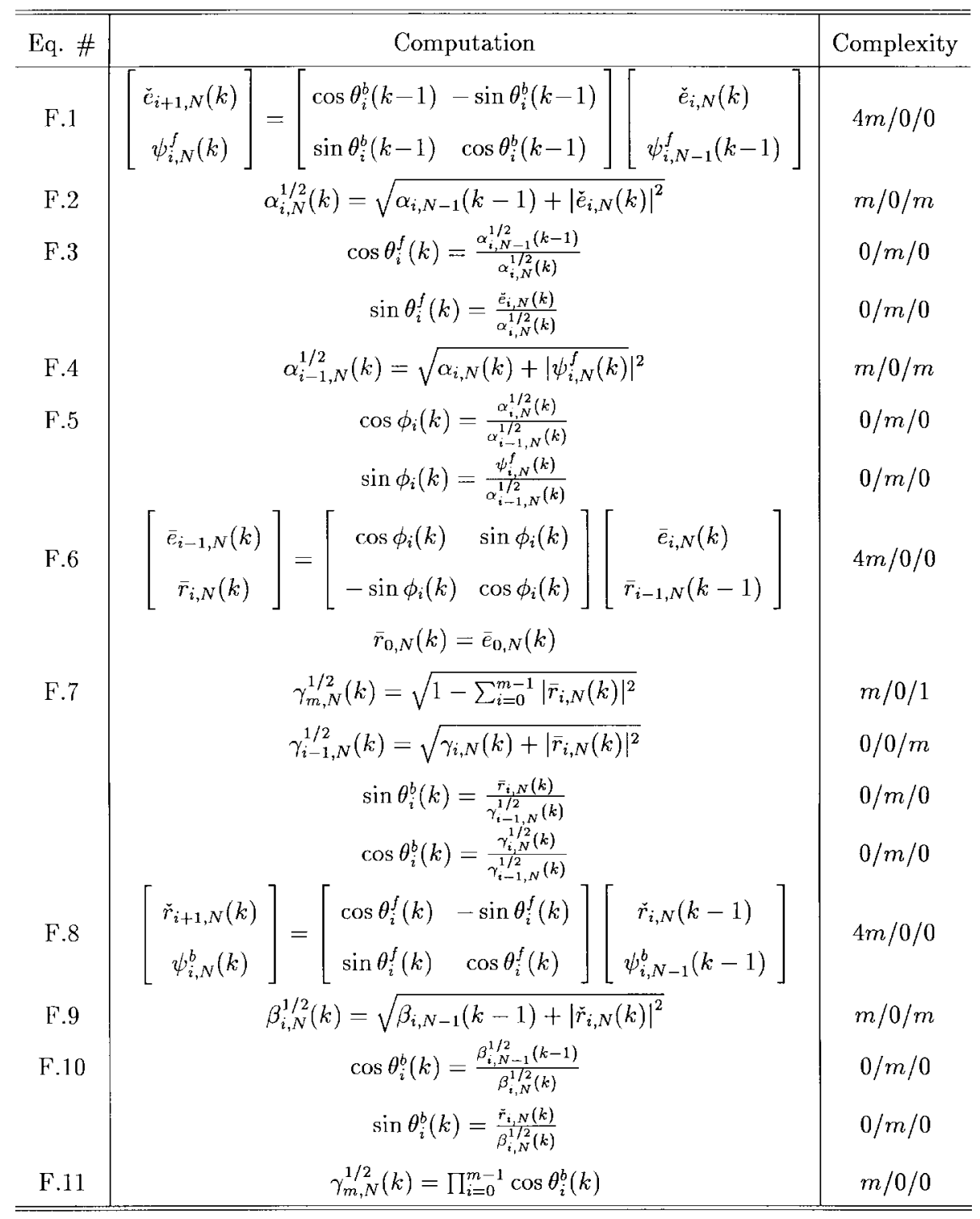

Note that the downdated forward prediction variables are not used to predict the rear edge, but the resulting updates from the previous section are incorporated since the window length is kept constant at $N$. Hence, (46), with reference to (41), is equivalent to

$$
\begin{aligned}
& {\left[\begin{array}{c}
\bar{a}_{i-1, N}(k) \\
\bar{b}_{i, N}(k)
\end{array}\right]} \\
& \quad=\left[\begin{array}{rr}
\cos \phi_{i}(k) & \sin \phi_{i}(k) \\
-\sin \phi_{i}(k) & \cos \phi_{i}(k)
\end{array}\right]\left[\begin{array}{c}
\bar{a}_{i, N}(k) \\
\bar{b}_{i-1, N}(k-1)
\end{array}\right]
\end{aligned}
$$

where, cf., Table I

$$
\begin{aligned}
& \bar{a}_{i, N}(k)=\frac{a_{i, N}(k)}{\alpha_{i, N}^{1 / 2}(k)}=\frac{\check{a}_{i, N}(k) \delta_{i, N}^{1 / 2}(k-1)}{\alpha_{i, N}^{1 / 2}(k)} \\
& \bar{b}_{i, N}(k)=\frac{b_{i, N}(k)}{\beta_{i, N}^{1 / 2}(k)}=\frac{\breve{b}_{i, N}(k) \delta_{i, N}^{1 / 2}(k)}{\beta_{i, N}^{1 / 2}(k)}
\end{aligned}
$$

for $i=m, \cdots, 1$ and $\bar{b}_{0, N}(k)=\bar{a}_{0, N}(k)$. The sequence of rotations start with

$$
\bar{a}_{m, N}(k)=\frac{\check{a}_{m, N}(k) \delta_{m, N}^{1 / 2}(k-1)}{\alpha_{m, N}^{1 / 2}(k)}
$$

The backward prediction variables $\bar{b}$ have an identical structure to (31) with sine-cosine pairs $\sin \vartheta^{b}$ and $\cos \vartheta^{b}$, as defined in (41). Thus, they can also be extracted similar to (39) with $\bar{b}, \delta^{1 / 2}$, and $\vartheta^{b}$ instead of $\bar{r}, \gamma^{1 / 2}$, and $\theta^{b}$, respectively.

Chambers' method can also be used in the fast QR algorithm for the window downdating of the forward prediction variables. Note that the number of hyperbolic rotations is less than the fast QR-lattice algorithm, and the downdated backward prediction variables are obtained via orthogonal rotations.

1) LINPACK Window Downdating Method: A third method for the downdating in the fast QR algorithm can also be 
TABLE III

Prediction of the Rear Edge of the Window

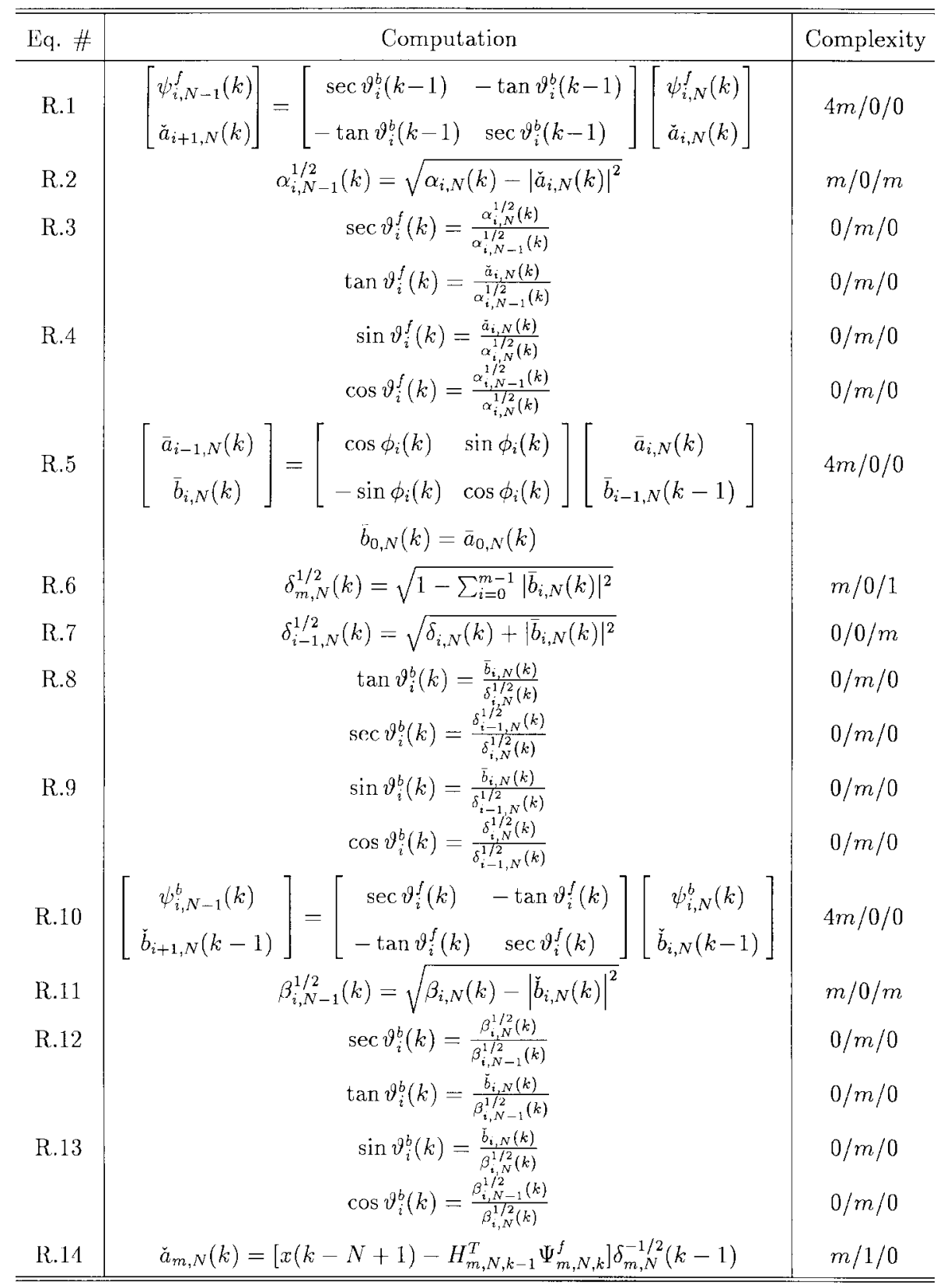

found by considering the following relation, cf., (15)

$$
\begin{aligned}
& {\left[\begin{array}{cc}
\Psi_{m, N, k}^{f} & \breve{R}_{m, N, k-1} \\
\check{a}_{m, N}(k) & \emptyset^{T}
\end{array}\right]} \\
& \quad=\boldsymbol{Q}_{m, N, k-1}^{d}\left[\begin{array}{cc}
\Psi_{m, N-1, k}^{f} & \breve{R}_{m, N-1, k-1} \\
x(k-N+1) & X_{m, k-N}^{T}
\end{array}\right] .
\end{aligned}
$$

The difference between $\boldsymbol{Q}^{d}$ matrices in (15) and (50) is that the rows of sole entry unity are deleted in $(50)$. The last column of the $\boldsymbol{Q}^{d}$ matrix is $\left[\begin{array}{ll}H_{m, N, k-1}^{T} & \delta_{m, N}^{1 / 2}(k-1)\end{array}\right]^{T}$, where $H$ is defined in (41). Since $\boldsymbol{Q}^{d}$ is an orthonormal matrix, $\left(Q^{d}\right)^{-1}=\left(Q^{d}\right)^{T}$, and hence, when the left-hand side of (50) is multiplied by $\left(Q^{d}\right)^{-1}$, we obtain the following relation by equating the lower left corner elements of both sides:

$$
x(k-N+1)=H_{m, N, k-1}^{T} \Psi_{m, N, k}^{f}+\delta_{m, N}^{1 / 2}(k-1) \check{a}_{m, N}(k)
$$

from which $\check{a}_{m, N}(k)$ can be solved and used as the initial condition for the orthogonal rotations given in (17). Note that the rotations that must be inverted do not destroy the circular nature of the computations. Hence, the downdated variables are obtained without hyperbolic rotations. This approach has its roots in [4].

\section{JOINT PROCESS EXTENSION}

Joint process extension in the fast QR and QR-lattice algorithms is identical to the forward prediction problem [18], [20] except the rotations are calculated by using the results 
TABLE IV

FAST QR-LATTICE Algorithm

\begin{tabular}{c|c||c|c}
\hline \hline Front edge equation & Complexity & Rear edge equation & Complexity \\
\hline F.1 & $4 m / 0 / 0$ & $R .1$ & $4 m / 0 / 0$ \\
F.2 & $m / 0 / m$ & $R .2$ & $m / 0 / m$ \\
F..3 & $0 / 2 m / 0$ & $R .3$ & $0 / 2 m / 0$ \\
F.8 & $4 m / 0 / 0$ & $R .10$ & $4 m / 0 / 0$ \\
F.9 & $m / 0 / m$ & $R .11$ & $m / 0 / m$ \\
F.10 & $0 / 2 m / 0$ & $R .12$ & $0 / 2 m / 0$ \\
F.11 & $m / 0 / 0$ & $(38)$ & $4 m / 0 / 0$ \\
(37) & $4 m / 0 / 0$ & R.4 (Chambers' Method) & $0 / 2 m / 0$ \\
(41) & $1 / 0 / 0$ & R.13(Chambers' Method) & $0 / 2 m / 0$ \\
\hline \multicolumn{4}{|c}{$29 m+1 / 8 m / 4 m$} \\
\hline
\end{tabular}

of the backward prediction of the input signal. Hence, we can obtain the relations

$$
\begin{aligned}
& {\left[\begin{array}{c}
\check{\epsilon}_{i+1, N}(k) \\
\psi_{i, N}(k)
\end{array}\right]} \\
& \quad=\left[\begin{array}{rr}
\cos \theta_{i}^{b}(k) & -\sin \theta_{i}^{b}(k) \\
\sin \theta_{i}^{b}(k) & \cos \theta_{i}^{b}(k)
\end{array}\right]\left[\begin{array}{c}
\breve{\epsilon}_{i, N}(k) \\
\psi_{i, N-1}(k-1)
\end{array}\right]
\end{aligned}
$$

for $i=0, \cdots, m-1$, where $\psi_{i}$ are the joint process extension variables. From the prediction of the rear edge of the window, we have

$$
\begin{aligned}
& {\left[\begin{array}{l}
\psi_{i, N-1}(k) \\
\check{v}_{i+1, N}(k)
\end{array}\right]} \\
& \quad=\left[\begin{array}{rr}
\sec \vartheta_{i}^{b}(k) & -\tan \vartheta_{i}^{b}(k) \\
-\tan \vartheta_{i}^{b}(k) & \sec \vartheta_{i}^{b}(k)
\end{array}\right]\left[\begin{array}{l}
\psi_{i, N}(k) \\
\check{v}_{i, N}(k)
\end{array}\right]
\end{aligned}
$$

for $i=0, \cdots, m-1$ similar to (22). Of course, the downdate can also be achieved by using the Chambers' or the LINPACK method. If (50) is modified accordingly

$$
\begin{aligned}
& {\left[\begin{array}{cc}
\Psi_{m, N, k} & \breve{\boldsymbol{R}}_{m, N, k} \\
\breve{v}_{m, N}(k) & \emptyset^{T}
\end{array}\right]} \\
& \quad=\boldsymbol{Q}_{m, N, k}^{d}\left[\begin{array}{cc}
\Psi_{m, N-1, k} & \breve{\boldsymbol{R}}_{m, N-1, k} \\
d(k-N+1) & X_{m, k-N}^{T}
\end{array}\right]
\end{aligned}
$$

where $\Psi$ is the vector of joint process extension variables, we can obtain the relation

$$
d(k-N+1)=H_{m, N, k}^{T} \Psi_{m, N, k}+\delta_{m, N}^{1 / 2}(k) \check{v}_{m, N}(k)
$$

from which $\breve{v}_{m, N}(k)$ can be solved. As the last step, the $a$ posteriori prediction residual is calculated. The computation is straightforward and achieved via

$$
\epsilon_{m, N}(k)=\gamma_{m, N}^{1 / 2}(k) \check{\epsilon}_{m, N}(k) .
$$

Tables IV-VI show the full listings of the fast QR and QR-lattice algorithms with reference to Tables II and III.

\begin{tabular}{|c|c|c|c|}
\hline Front edge equation & Complexity & Rear edge equation & Complexity \\
\hline F.1 & $4 m / 0 / 0$ & R. 1 & $4 m / 0 / 0$ \\
\hline $\mathrm{F} .2($ for $i=m)$ & $1 / 0 / 1$ & R.2 (for $i=m)$ & $1 / 0 / 1$ \\
\hline F.4 & $m / 0 / m$ & R.5 & $4 m / 0 / 0$ \\
\hline F.5 & $0 / 2 m / 0$ & R. 6 & $m / 0 / 1$ \\
\hline F.6 & $1 m / 0 / 0$ & R.7 & $0 / 0 / m$ \\
\hline F.7 & $m / 2 m / m+1$ & R. 8 & $0 / 2 m / 0$ \\
\hline (37) & $1 m / 0 / 0$ & $(38)$ & $4 \mathrm{~m} / 0 / 0$ \\
\hline (41) & $1 / 0 / 0$ & R.9 (Chambers' Method) & $0 / 2 m / 0$ \\
\hline \multicolumn{4}{|c|}{$8 m / 3 m+4$ (Chambers' Method) } \\
\hline
\end{tabular}

\section{SimULATIONS}

The fast QR and fast QR-lattice algorithms are simulated to reveal the numerical properties for different wordlengths on a floating-point processor. The full sliding window QR (nonfast) algorithm is also included. The experiment with sinusoidal
TABLE V

FAST QR ALGORITHM

TABLE VI

FAST QR ALGORITHM WITH LINPACK Downdating

\begin{tabular}{c|c}
\hline Rear edge equation & Complexity \\
\hline R.14 & $m / 1 / 0$ \\
Invert (14) & $4 m / 0 / 0$ \\
R.2 (for $i=m$ ) & $1 / 0 / 1$ \\
R.5 & $4 m / 0 / 0$ \\
R.6 & $m / 0 / 1$ \\
R.7 & $0 / 0 / m$ \\
R..9 & $0 / 2 m / 0$ \\
Modify R.14 as in (40) & $m / 1 / 0$ \\
Modify (14) for JPE and invert & $4 m / 0 / 0$ \\
\hline \multicolumn{2}{|c}{ Total: $29 m+3 / 6 m / 3 m+4$} \\
\hline \hline
\end{tabular}

inputs from [18] is repeated. The input signal to the adaptive filter is

$$
x(k)=\cos (0.05 \pi k)+\sqrt{2} \cos (0.3 \pi k)+n(k)
$$

where $n(k)$ is white noise with variance $10^{-10}$. The signal is used to identify a reference system of length 8 . The $8 \times 8$ covariance matrix of this signal is nearly singular, its rank being mildly displaced from 4 by four eigenvalues at $10^{-10}$. Such a signal can easily reveal the numerical problems of adaptive algorithms. The mean squared error of the adaptive algorithms are presented in Figs. 1-3 for different precisions. The window size is 20 samples; $\alpha$ and $\beta$ are initialized to $10^{-3}$. All three algorithms perform well for double precision (53 bit mantissa) implementation, and in the long run (billions of samples), we have not observed numerical problems. As the number of bits is decreased in the mantissa, the algorithms have difficulty maintaining stability. The QRlattice algorithm is prone to quantization; the Chambers' method helps to some extent. The fast $\mathrm{QR}$ algorithm is more immune to quantization, but the Chambers' and LINPACK methods worsen the numerical conditioning of the fast QR algorithm. This may be due to the fact that more variables are quantized. The full QR algorithm, which does not rely on exact relationships between forward and backward prediction variables, can maintain the stability even for a 28-bit mantissa. The Chambers' and LINPACK methods may contribute to amplification of numerical errors unless the wordlength is 


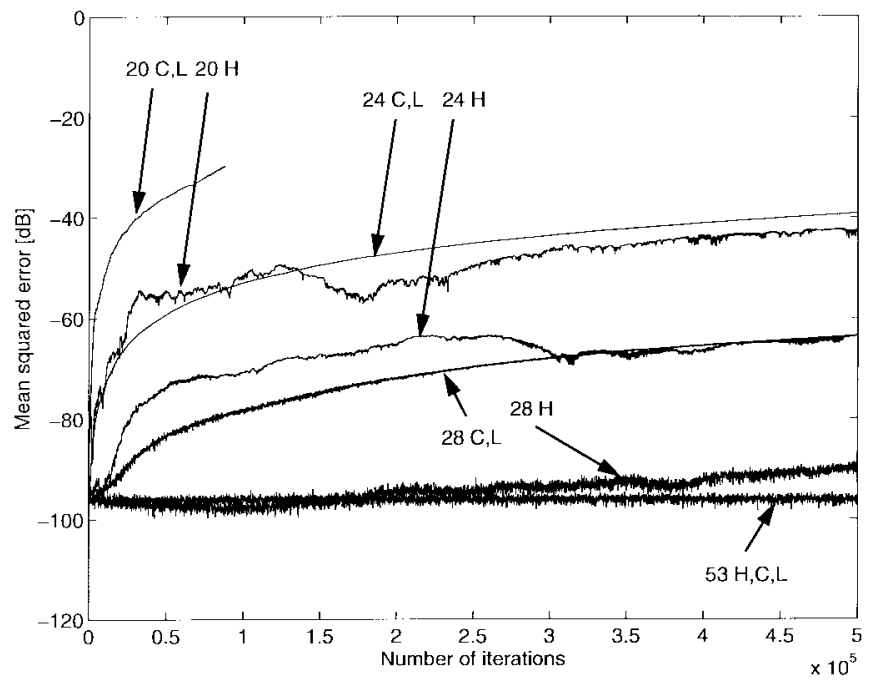

Fig. 1. Comparison of the fast $\mathrm{QR}$ algorithm for different implementations and number of bits in the mantissa. H: Hyperbolic rotations. C: Chambers' method. L: LINPACK downdating.

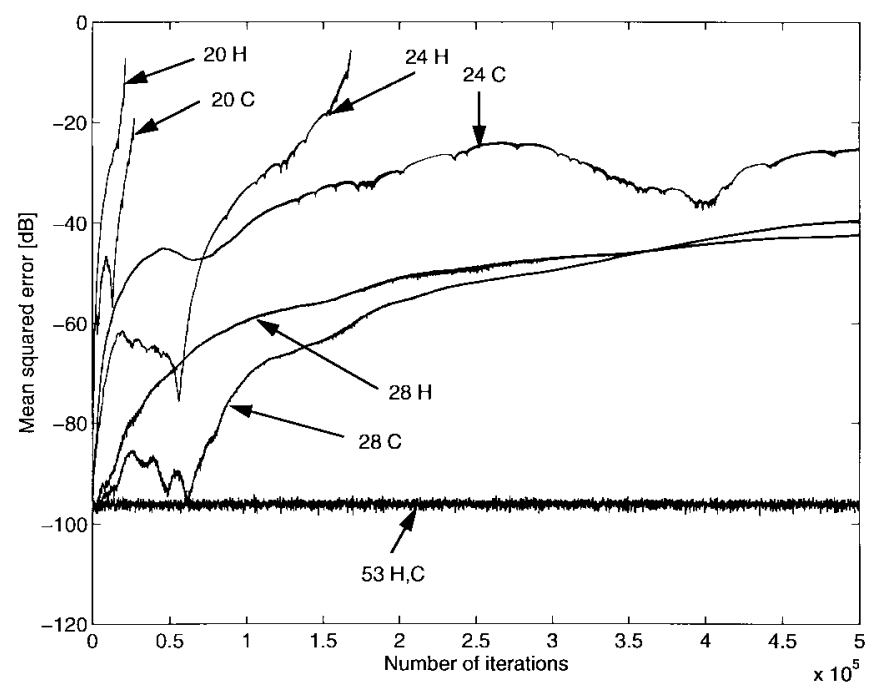

Fig. 2. Comparison of the fast QR-lattice algorithm for different implementations and number of bits in the mantissa. H: Hyperbolic rotations. C: Chambers' method.

sufficiently long. In summary, the sliding window QR methods can be practically regarded as "stable" for long wordlengths.

In an other experiment, we have simulated the algorithms in an acoustic echo cancellation setup. The simulation signals are real recordings with a dynamic range of $\mp 1.33 \times 10^{4}$. We have used the double-precision mantissa (53 bits) for computations. The input is a speech signal of duration 500000 samples $(62.5 \mathrm{~s})$. This setup is identical to a classical system identification problem. The unknown system has 64 taps, and the window length is chosen as 128 . The SNR level of the desired signal is approximately $25 \mathrm{~dB}$. As a figure of merit, we have calculated and compared the residual mean square echo [mean square error (MSE)] power of all algorithms over the full simulation. It is observed that all algorithms have almost identical performance with no apparent numerical problems; they differ only in fractions of the order $10^{3}$ to $10^{-10}$. The MSE is approximately $2.2 \times 10^{4}$.

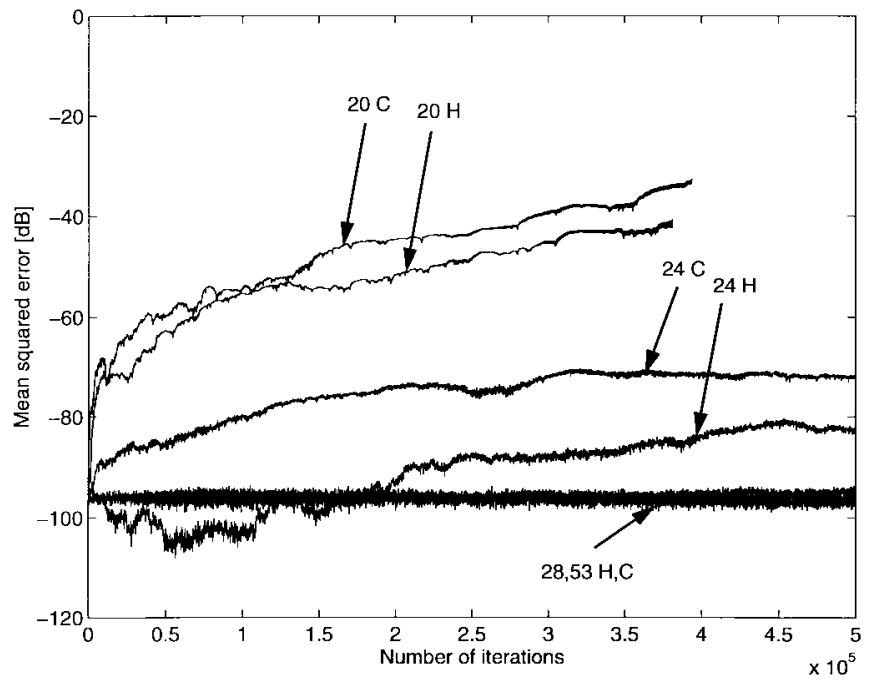

Fig. 3. Comparison of the full QR (nonfast) algorithm for different implementations and number of bits in the mantissa. H: Hyperbolic rotations. C: Chambers' method.

\section{CONCLUDING REMARKS}

The theme of this paper has been to generalize the existing fast QR algorithms to incorporate sliding windows. Various methods are proposed for the delicate step of window downdating. It is observed that sliding window QR algorithms can be regarded as practically stable; as for the double-precision applications, the algorithms maintain stability over billions of iterations. The numerical properties of the sliding window techniques have yet to be proved theoretically.

The presentation has followed the lines of classical QR decomposition with Givens rotations and square roots. Further elaboration on how to remove square roots and find more computationally efficient structures should be rather straightforward after this stage. Indeed, the transformations and variables can be modified [19], [24] to yield alternative implementations. The enhanced numerical properties of the square-root-free implementations [19] are also expected to reflect to sliding window formulations.

\section{ACKNOWLEDGMENT}

The authors would like to thank the anonymous reviewers for their constructive comments, which helped improve the presentation.

\section{REFERENCES}

[1] J. Cioffi and T. Kailath, "Windowed fast transversal filters adaptive algorithms with normalization," IEEE Trans. Acoust., Speech, Signal Processing, vol. ASSP-33, pp. 607-624, 1985.

[2] J. Cioffi, "Limited-precision effects in adaptive filtering," IEEE Trans. Circuits Syst., vol. CAS-34, pp. 821-833, July 1987.

[3] J. Chambers, "Regression updating," J. Amer. Stat. Assoc., vol. 66, pp. 744-748, 1971.

[4] P. Gill, G. Golub, W. Murray, and M. Saunders, "Methods of modifying matrix factorizations," Math. Comput., vol. 28, pp. 505-535, 1974.

[5] G. Stewart, "On the stability of sequential updates and downdates," IEEE Trans. Signal Processing, vol. 43, pp. 2642-2648, Nov. 1995.

[6] D. Slock, "The backward consistency concept and roundoff error propagation dynamics in recursive least-squares algorithms," Opt. Eng., vol. 31, pp. 1153-1169, 1992. 
[7] P. Regalia, "Numerical stability properties of a QR-based fast leastsquares algorithm," IEEE Trans. Signal Processing, vol. 41, pp 2096-2109, June 1993

[8] _ "Past input reconstruction in fast least-squares algorithms," IEEE Trans. Signal Processing, vol. 45, pp. 2231-2240, Sept. 1997.

[9] B. Porat, B. Friedlander, and M. Morf, "Square root covariance ladder algorithms," IEEE Trans. Automat. Contr., vol. AC-27, pp. 813-829, 1982.

[10] K. Zhao, F. Ling, H. Levari, and J. Proakis, "Sliding window orderrecursive least-squares algorithms," IEEE Trans. Signal Processing, vol. 42, pp. 1961-1972, Aug. 1994.

[11] S. F. Hsieh, K. J. Liu, and K. Yao, "Dual-state systolic architectures for up/downdating RLS adaptive filtering," IEEE Trans. Circuits Syst. II, vol. 39, pp. 382-385, June 1992.

[12] M. Mahon, L. Sibul, and H. Valenzuela, "A sliding window update for the basis matrix of the QR-decomposition," IEEE Trans. Signal Processing, vol. 41, pp. 1951-1953, May 1993.

[13] P. Strobach and D. Goryn, "A computation of the sliding window recursive QR decompostion," in Proc. ICASSP, 1993, pp. 29-32.

[14] K. Yoo and H. Park, "Fast residual computation for sliding window recursive least-squares," Signal Process., vol. 45, pp. 85-95, 1995.

[15] A. Bjorck, H. Park, and L. Elden, "Accurate downdating of least-squares solutions," SIAM J. Matrix Anal. Appl., vol. 15, no. 2, pp. 549-568, 1994.

[16] A. Bojanczyk, R. Brent, P. Van Dooren, and F. De Hoog, "A note on downdating the Cholesky factorization," SIAM J. Sci. Stat. Comput., vol. 8, pp. 210-221, May 1987.

[17] J. Cioffi, "The fast adaptive ROTOR's algorithm," IEEE Trans. Acoust., Speech, Signal Processing, vol. 38, pp. 631-653, Apr. 1990.

[18] P. Regalia and M. Bellanger, "On the duality between fast QR methods and lattice methods in least squares adaptive filtering," IEEE Trans. Signal Processing, vol. 39, pp. 879-891, Apr. 1991.

[19] I. Proudler, J. McWhirter, and T. Shepherd, "Computationally efficient QR decomposition approach to least squares adaptive filtering," Proc. Inst. Elect. Eng. pt. F, vol. 138, pp. 341-353, Aug. 1991.

[20] F. Ling, "Givens rotation based least-squares lattice and related algorithms," IEEE Trans. Signal Processing, vol. 39, pp. 1541-1551, July 1991.

[21] F. Ling, D. Manolakis, and J. Proakis, "A recursive modified Gram-Schmidt algorithm for least-squares estimation," IEEE Trans. Acoust., Speech, Signal Processing, vol. ASSP-34, pp. 829-836, Apr. 1986.

[22] S. Haykin, Adaptive Filter Theory, 2nd ed. Englewood Cliffs, NJ: Prentice-Hall, 1991

[23] M. Bellanger, "A survey of QR based fast least squares adaptive filters: From principles to realization," in Proc. ICASSP, 1991, pp. 1833-1836.

[24] S. F. Hsieh, K. J. Liu, and K. Yao, "A unified square-root-free approach for QRD-based recursive least squares estimation," IEEE Trans. Signal Processing, vol. 41, pp. 1405-1409, Mar. 1993.

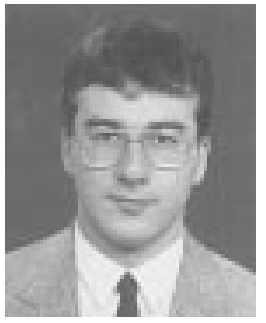

Buyurman Baykal (M'93) was born in Ankara, Turkey in 1968. He received the B.Sc. degree (High Hons.) from the Middle East Technical University, Ankara, in 1990 and the MS.c. (Distinction) and the $\mathrm{Ph} . \mathrm{D}$. degrees from the Imperial College of Science, Technology, and Medicine, University of London, London, U.K., in 1992 and 1995, respectively, all in electrical and electronic engineering.

He held the British Council fellowship from 1991 to 1992 for postgraduate studies in the U.K. From 1993 to 1997 , he was employed by the Imperial College, where he was involved with various research projects on echo cancellation, noise reduction, and channel equalization. He is currently working as an Assistant Professor with Başkent University, Ankara. His research interests include adaptive systems and signal processing in the areas of telecommunications, wireless, and broadcast technology.

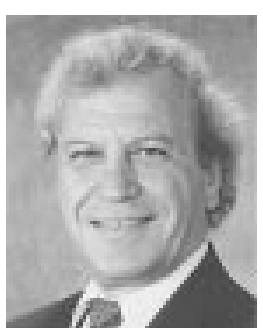

Anthony G. Constantinides (SM'78) is Professor of Signal Processing and the Head of the Signal Processing and Digital Systems Section, Department of Electrical and Electronic Engineering, Imperial College of Science, Technology, and Medicine, University of London, London, U.K. He has been actively involved with research in various aspects of digital filter design, digital signal processing, and communications for a number of years. His research spans a wide range of digital signal processing, both from the theoretical as well as the practical points of view. His recent work has been directed toward the demanding signal processing problems arising from the area of telecommunications. He has published a range of books and papers in learned journals in the area of digital signal processing and its applications.

Dr. Constantinides served as the first President of the European Association for Signal Processing (EURASIP) and has contributed in this capacity to the establishment of the European Journal for Signal Processing. He has been on, and is currently serving as, a member of many Technical Program Committees of IEEE international conferences. He organized the first ever international series of meetings on digital signal processing in London, initially in 1967 , and in Florence (with V. Cappellini) since 1972. In 1985, he was awarded the Honor of Chevalier, Palmes Academiques, by the French government, and in 1996, he was promoted to Officer, Palmes Academiques. He holds several visiting professorships and other fellowships and honors around the world. He presently is serving as a member of the Signal Processing Society Technical Committee on Neural Networks for Signal Processing of the IEEE Signal Processing Society. 